\title{
Induction of Cyclooxygenase-2 in Human Umbilical Vein Endothelial Cells by Lysophosphatidylcholine
}

\author{
Artur Zembowicz, Stephen L. Jones, and Kenneth K. Wu \\ Vascular Biology Research Center and Division of Hematology, Department of Internal Medicine, The University of Texas \\ Houston Health Science Center, Houston, Texas 77030
}

\begin{abstract}
Lysophosphatidylcholine (lysoPC), a component of atherogenic lipoproteins and atherosclerotic lesions, has been recently suggested to play a role in atherogenesis. LysoPC is known to induce several endothelial genes involved in leukocyte recruitment, mitogenesis, and inflammation. Cyclooxygenases (prostaglandin $\mathrm{H}_{2}$ synthases) are rate-limiting enzymes involved in the endothelial synthesis of prostacyclin, an antiplatelet, vasorelaxant, and vasoprotective molecule. We investigated the effect of lysoPC on the endothelial expression of cyclooxygenases. Our results demonstrate that, in cultured human umbilical vein endothelial cells, lysoPC induces cyclooxygenase-2 mRNA and protein levels. Increased expression of cyclooxygenase- 2 is accompanied by the enhancement of both basal- and calcium ionophore A23187-induced synthesis of prostacyclin. Nuclear runoff experiments demonstrated an increased rate of transcription of the cyclooxygenase- 2 gene by lysoPC. In contrast, lysoPC did not affect the expression of constitutive cyclooxygenase-1. Our results suggest that the induction of endothelial cyclooxygenase- 2 by lysoPC may be an important vasoprotective mechanism that limits progression of atherosclerotic lesions and promotes their regression. (J. Clin. Invest. 1995. 96:1688-1692.) Key words: nitric oxide • prostacyclin • endothelium - atherosclerosis - low density lipoproteins
\end{abstract}

\section{Introduction}

Cyclooxygenases (COX, ${ }^{1}$ prostaglandin $\mathrm{H}_{2}$ synthases) are key rate-limiting enzymes in the synthesis of prostanoids from arachidonic acid (1). Two isoforms of COX have been described

Address correspondence to Artur Zembowicz, Vascular Biology $\mathrm{Re}$ search Center and Division of Hematology, Department of Internal Medicine, University of Texas Houston Health Science Center, 6431 Fannin, Houston, TX 77030. Phone: 713-792-5450; FAX: 713-794-4230; E-mail: azemb@heart.med.uth.tmc.edu

Received for publication 25 April 1995 and accepted in revised form 31 May 1995.

1. Abbreviations used in this paper: COX, cyclooxygenase; GAPDH, glyceraldehyde-3-phosphate dehydrogenase; HUVEC, human umbilical vein endothelial cells; lysoPC, lysophosphatidylcholine.

J. Clin. Invest.

(C) The American Society for Clinical Investigation, Inc.

0021-9738/95/09/1688/05 \$2.00

Volume 96, September 1995, 1688-1692 so far (2-6). COX-1 is constitutively expressed in many cell types. In contrast, expression of COX-2 requires stimulation of cells with cell-specific extracellular signals such as autacoids, hormones, growth factors, and cytokines $(7,8)$. Both isoforms of COX have been reported to be expressed in human umbilical vein endothelial cells (HUVEC) (9-12).

The major prostanoid synthesized by vascular endothelium is prostacyclin $\left(\mathrm{PGI}_{2}\right)(13)$ which regulates various physiological processes occurring at the interphase between the blood and vascular wall. Biological actions of prostacylin include inhibition of platelet aggregation and adhesion, inhibition of vascular smooth muscle tone and growth, inhibition of leukocyte activation and adhesion, and reduction of cholesteryl ester accumulation in cells of the vessel wall (14). These biological actions of prostacyclin and its efficacy in the treatment of complications of atherosclerosis such as peripheral vascular disease $(15,16)$ suggest that prostacylin is an endogenous antiatherogenic molecule.

Several lines of evidence suggest a role for lysophosphatidylcholine (lysoPC) in atherogenesis (17). The lysoPC content of atherosclerotic arteries is severalfold higher than that of normal vessels (18). After proatherogenic modifications of LDLs, lysoPC may constitute up to $40 \%$ of their total lipid content (19). LysoPC is an essential component of oxidized proatherogenic lipoproteins responsible for their chemotactic effect on human monocytes $(20,21)$ and mitogenic effect on murine macrophages (22). LysoPC induces monocytic cell expression of heparin-binding epidermal growth factor (23). Importantly, lysoPC causes induction of several endothelial genes expressed in early atherosclerosis, such as vascular adhesion molecule-1, intercellular adhesion molecule-1, PDGF chains A and B, and heparinbinding EGF $(24,25)$.

Surprisingly, we have recently demonstrated that lysoPC augments the endothelial expression of nitric oxide synthase type III which is believed to be a key vasoprotective and antiatherogenic enzyme (25a). Prostacyclin and nitric oxide have many complementary or synergistic vasoprotective actions and, hence, are believed to serve similar biological functions. Therefore, to determine whether lysoPC is also involved in the regulation of vasoprotective molecules other then nitric oxide, we investigated the effects of lysoPC on the expression of COX isoforms in human umbilical vein endothelial cells. The results presented in this communication demonstrate that lysoPC induces expression of COX-2 with resultant augmentation of prostacyclin synthesis.

\section{Methods}

Materials. LysoPC (1-palmitoyl-2-hydroxy-sn-glycero-3-phosphocholine) was from Avanti Polar Lipids (Alabaster, AL). Unless otherwise 
indicated all the other reagents were from Sigma Chemical Co. (St. Louis, MO).

Tissue culture. HUVEC were cultured as described previously (26) in medium 199 containing 20\% bovine calf serum (Hyclone Laboratories, Logan, UT), $12.5 \mu \mathrm{g} / \mathrm{ml}$ endothelium cell mitogen (Biomedical Technologies, Inc., Stoughton, MA) and $100 \mu \mathrm{g} / \mathrm{ml}$ heparin. Only second and third passage cells were used. $12 \mathrm{~h}$ before lysoPC treatment, cells were transferred to medium 199 containing 5\% FBS and no other additives.

Northern blotting. Total RNA was isolated using Ultraspec (Biotecx Laboratories, Houston, TX). 15-25 $\mu \mathrm{g}$ of RNA was fractionated on 1\% agarose and transferred to a positively charged nylon membrane. As COX-1 probe we used agarose gel-purified full-length COX-1 cDNA cloned in our laboratory (11). As COX-2 probe we used agarose gelpurified full-length 1.94-kb COX-2 cDNA which was obtained from Dr. Timothy Hla (9). Probe labeling, hybridization, and chemiluminescent detection were performed using components of Genius system (Boehringer Mannheim Corp., Indianapolis, IN). Membranes were hybridized overnight in $5 \times$ SSC containing $0.02 \%$ SDS, $0.1 \%$ sarcosyl, and $2 \%$ blocking reagent (Boehringer Mannheim) at $68^{\circ} \mathrm{C}$. Nonspecifically bound probe was washed during $45 \mathrm{~min}$ of high stringency washes performed at $60^{\circ} \mathrm{C}$ in $0.1 \times$ SSC containing $0.1 \%$ SDS. Membranes were stripped by boiling in $0.2 \times$ SSC containing $1 \%$ SDS for $15 \mathrm{~min}$ and rehybridized to a digoxigenin-labeled glyceraldehyde-3-phosphate dehydrogenase (GAPDH) cDNA probe. Blots were quantitated using the Bio Image system (MilliGen, Burlington, MA).

Western blotting. After incubation with lysoPC for indicated time HUVEC were washed twice in ice-cold PBS and lysed in boiling buffer containing Tris $\mathrm{HCl}(0.05 \mathrm{M}, \mathrm{pH} 7.4)$ and 1\% SDS. Protein concentration in lysates was determined using DC protein assay (Bio-Rad Laboratories, Richmond, CA). Lysates (15 $\mu \mathrm{g}$ protein) were electrophoresed on $10 \% \mathrm{SDS} /$ polyacrylamide gel and electroblotted onto a nitrocellulose membrane. The COX proteins on the membranes were detected using rabbit anti-ram seminal vesicles COX-1 polyclonal antibody raised in our laboratory or rabbit anti-human COX-2 polyclonal antibody specific for COOH-terminal insert of COX-2 obtained from Dr. P. Marshall (Ciba-Geigy, $\mathrm{NJ}$ ) and visualized using enhanced chemiluminescence system (Amersham Corp., Arlington Heights, IL).

Nuclear runoff experiments. Isolation of nuclei and synthesis of labeled RNA were performed according to a standard laboratory procedure (27). HUVEC nuclei $\left(10^{7}\right)$ were incubated in the presence of 0.25 $\mathrm{mCi}$ of $\left[{ }^{32} \mathrm{P}\right] \mathrm{GTP}$ and other unlabeled nucleotides $(1 \mathrm{mM})$ at $26^{\circ} \mathrm{C}$ in a vol of $200 \mu \mathrm{l}$ for $25 \mathrm{~min}$. Transcribed RNAs were isolated using Ultraspec and equal amounts $\left(6-8 \times 10^{6} \mathrm{cpm}\right)$ were hybridized to denatured plasmids $(15 \mu \mathrm{g})$ containing cDNAs for COX-1 (in pSG5 vector; Stratagene Inc., La Jolla, CA), COX-2 (in pVL1393; Pharmingen, San Diego, CA), and vWF (in Bluescript; Stratagene) and hamster $\beta$-tubulin (in pAcUW51; Pharmingen) which were immobilized on nitrocellulose membranes. Conditions for hybridization and washes were identical to those during Northern blotting.

Prostacyclin synthesis. HUVEC were incubated with lysoPC for 12 h. Afterwards, cells were washed twice in warm $\left(37^{\circ} \mathrm{C}\right) 10 \mathrm{mM}$ Hepes buffer (pH 7.4) containing $2.5 \mathrm{mM} \mathrm{CaCl}_{2}, 1 \mathrm{mM} \mathrm{MgCl}_{2}, 5 \mathrm{mM} \mathrm{KCl}$, and $145 \mathrm{mM} \mathrm{NaCl}$ and incubated in $10 \mathrm{ml}$ of the same buffer for 20 min. To determine the effect of lysoPC on prostacyclin synthesis by activated HUVEC, calcium ionophore A23187 (3 $\mu \mathrm{M})$ was added to some of the flasks during the last $10 \mathrm{~min}$ of incubation. The concentration of 6-keto $\mathrm{PGF}_{1 \alpha}$, a stable hydrolysis product of prostacyclin, was measured in cell supernatants by an RIA (28).

\section{Results}

In the presence of $5 \%$ serum, lysoPC at concentrations of 100 $\mu \mathrm{M}$ and below did not affect the viability of HUVEC monolayers, as estimated by trypan blue exclusion and cell counting. HUVEC could be cultured in the presence of lysoPC for at least up to $80 \mathrm{~h}$ without any signs of cytotoxicity. LysoPC at the concentration of $100 \mu \mathrm{M}$ did not significantly affect the levels
A

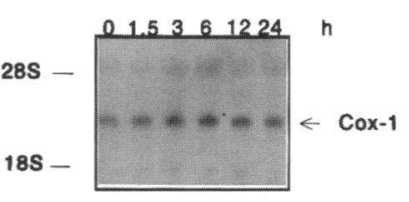

Figure 1. Effect of lysoPC on mRNA levels of COX-1 and COX-2 in HUVEC. $(A)$ Northern blotting analysis of $25 \mu \mathrm{g}$ of total RNA isolated from control HUVEC (lane 0 ) and HUVEC stimulated with LysoPC $(100 \mu \mathrm{M})$ for $1.5,3,6$, 12 , and 24 h using digoxigenin-labeled COX-1 cDNA probe. (B) Autoradiogram of the same membrane as in $A$ after stripping and rehybridization using digoxigenin-labeled COX-2 probe. (C) Autoradiogram of the same membrane as in $A$ and $B$ after stripping and rehybridization using digoxigenin-labeled GAPDH probe. These experiments were repeated twice with similar results.

of 2.8-kb COX-1 mRNA in cultured HUVEC, as determined by Northern blotting (Fig. $1 \mathrm{~A}$ ). Densitometric analysis revealed that lysoPC-induced increases of COX-1 mRNA levels were $<1.5$-fold. In contrast, lysoPC $(100 \mu \mathrm{M})$ caused a marked timedependent induction of $\sim 4-4.5-\mathrm{kb}$ COX-2 mRNA (Fig. $1 B$ ). COX-2 mRNA levels reached a maximum $3 \mathrm{~h}$ after the stimulation with lysoPC. Interestingly, in addition to 4-4.5-kb COX2 mRNA, our COX-2 probe hybridized to a distinct $2.8-\mathrm{kb}$ mRNA (Fig. $1 B$ ). Despite a similar size as that of COX-1 mRNA, this $2.8-\mathrm{kb}$ band is unlikely to be a result of crosshybridization of our COX-2 probe to COX-1 mRNA because this mRNA species is not present in unstimulated HUVEC. Moreover, this $2.8-\mathrm{kb}$ mRNA was clearly inducible by lysoPC with a time-course identical to that of induction of COX-2. This suggests that this $2.8-\mathrm{kb}$ mRNA species is related to COX-2.

Western blotting analysis revealed that the induction of COX-2 mRNA by lysoPC was paralleled by the appearance of COX-2 protein (Fig. 2). COX-2 protein level reached a peak 3 $\mathrm{h}$ after stimulation with lysoPC $(100 \mu \mathrm{M})$ and remained detectable for at least $24 \mathrm{~h}$ after stimulation of HUVEC with lysoPC. The lowest concentration of lysoPC that was able to induce COX-2 protein expression after $3 \mathrm{~h}$ of stimulation with lysoPC was $50 \mu \mathrm{M}$. LysoPC at $30 \mu \mathrm{M}$ was without effect $(n=2$, not shown). As shown in Fig. 2, the antibody used for detection of COX-2 did not cross-react with COX-1. Western blotting analysis of HUVEC lysates using anti-COX-1 antibody revealed the presence of a $72 / 74-\mathrm{kD}$ doublet. The higher molecular weight band was clearly inducible by lysoPC with a time-course similar to that of induction of COX-2. The lower molecular weight band did not change after stimulation with lysoPC. These results raised suspicions as to the isoform specificity of our COX-1 antibody. Indeed, as shown in Fig. 2, the COX-1 antibody crossreacted with COX-2. Therefore, it is likely that the higher molecular weight band of the doublet in Fig. $2 \mathrm{~A}$ may represent mainly lysoPC-inducible COX-2 and the lower molecular weight band of the doublet may represent mainly noninducible COX-1.

To establish whether induction of COX-2 by lysoPC is due to the activation of the COX-2 gene we performed nuclear runoff experiments. As seen in Fig. 3, nuclei isolated from HUVEC $3 \mathrm{~h}$ after stimulation with lysoPC synthesized a significantly greater amount of COX-2 mRNA than the control HUVEC. In contrast, the rate of transcription of the COX-1 
A

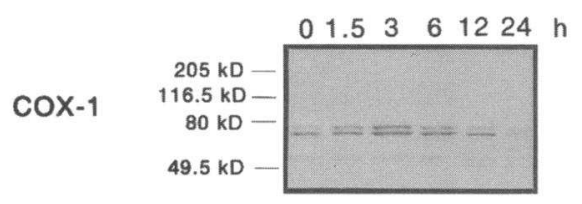

$\mathbf{B}$

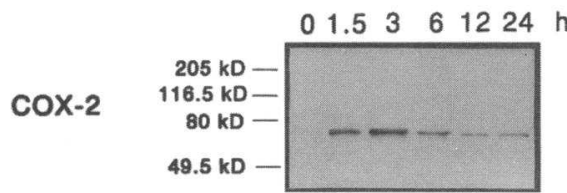

Figure 2. Effect of lysoPC on levels of COX-1 and COX-2 protein in HUVEC. $(A)$ Western blotting analysis of COX-1 in lysates prepared from control HUVEC (lane 0 ) and those prepared from HUVEC stimulated with lysoPC $(100 \mu \mathrm{M})$ for $1.5,3,6,12$, and $24 \mathrm{~h}$. (Inset) Western blotting analysis of $1 \mu \mathrm{g}$ of COX-1 standard ( $1 \mu \mathrm{g}$ of lysate of Cos-1 cells transiently transfected with human COX-1) and COX-2 standard ( $1 \mu \mathrm{g}$ of lysate of Sf9 cells expressing human COX-2 in a baculovirus system). This demonstrates that anti-COX-1 antibody is binding to both isoforms of COX. $(B)$ Western blotting analysis of COX-2 in lysates prepared from control HUVEC (lane 0 ) and those prepared from HUVEC stimulated with lysoPC $(100 \mu \mathrm{M})$ for $1.5,3,6,12$, and $24 \mathrm{~h}$. (Inset) Western blotting analysis of COX-1 and COX-2 standards. This demonstrates that anti-COX-2 antibody is specific for COX-2. This experiment was repeated four times with similar results.

gene was not affected by lysoPC. In the same experiments we did not observe any lysoPC-induced changes in the transcription rate of vWF (Fig. 3) and $\beta$-tubulin or GAPDH genes (data not shown).

To establish whether induction of COX-2 in HUVEC has the expected functional consequence for the endothelial synthesis of prostacyclin, we determined the production of 6-keto-PGF $\mathrm{Pa}_{1 \alpha}$, a stable product of hydrolysis of prostacyclin, by control and lysoPC-stimulated HUVEC. As shown on Fig. 4, incubation of HUVEC for $12 \mathrm{~h}$ with lysoPC $(100 \mu \mathrm{M})$ approximately doubled basal prostacyclin synthesis whereas prostacyclin release in response to calcium ionophore A23187 was increased about fivefold.

\section{Discussion}

The major finding of this communication is demonstration of the induction of COX-2 in cultured human umbilical vein endo-

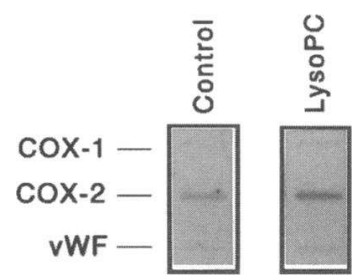

Figure 3. Nuclear runoff analysis of transcription rates of $\mathrm{COX}-1, \mathrm{COX}-2$, and $\mathrm{vWf}$ genes in control HUVEC and in HUVEC stimulated with lysoPC $(100 \mu \mathrm{M})$ for $3 \mathrm{~h}$. Autoradiogram shows amounts of radiolabeled RNA synthesized by nuclei isolated from control and lysoPC-stimulated (100 $\mu \mathrm{M}, 3 \mathrm{~h})$ HUVEC that hybridized to plasmids containing cDNAs of human COX-1, COX-2, and vWf. This experiment was performed four times with similar results. Densitometric analysis of bands revealed that hybridization of RNA synthesized by lysoPCstimulated HUVEC to COX-2 cDNA was $3.9 \pm 0.3$-fold higher $(n=4)$ that hybridization of RNA synthesized by control cells.

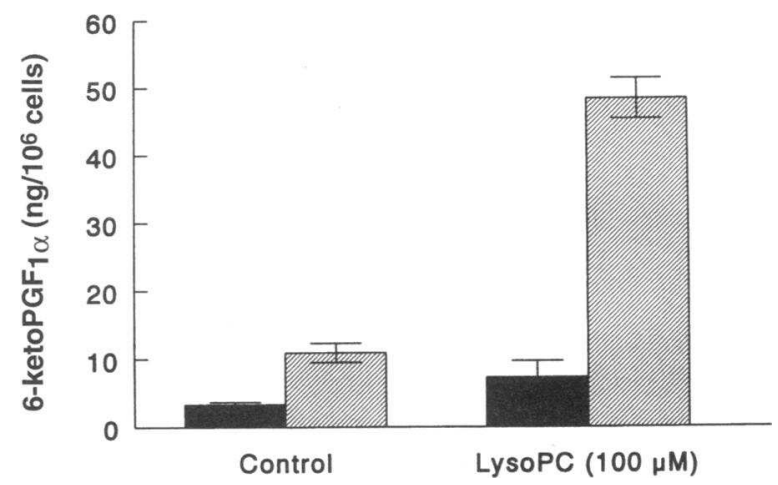

Figure 4. Enhancement of prostacyclin synthesis in HUVEC by lysophosphatidylcholine. Graph shows production of 6-ketoPGF ${ }_{1 \alpha}$, a stable metabolite of prostacyclin, by HUVEC monolayers cultured in the absence (Control) and in the presence of lysoPC $(100 \mu \mathrm{M})$ for $12 \mathrm{~h}$. Experimental details are described in Methods. Solid bars represent synthesis of 6ketoPGF $F_{1 \alpha}$ by unstimulated HUVEC. Hatched bars represent synthesis of 6-ketoPGF ${ }_{1 \alpha}$ in the presence of calcium ionophore A23187 (3 $\left.\mu \mathrm{M}\right)$. Bars represent means \pm SE of $n=4$ experiments.

thelial cells by lysoPC. In the presence of 5\% serum, lysoPC, at nontoxic concentrations of $50-100 \mu \mathrm{M}$, caused a rapid induction of COX-2 mRNA and protein levels. In contrast, lysoPC did not affect COX-1 mRNA or protein levels. Interestingly, in addition to 4-4.5-kb COX-2 mRNA, our COX-2 probe hybridized to a distinct 2.8-kb mRNA (Fig. $1 B$ ). Despite a similar size as that of COX-1 mRNA, this $2.8-\mathrm{kb}$ band is unlikely to be a result of cross-hybridization of our COX-2 probe to COX1 mRNA because this mRNA species is not present in unstimulated HUVEC. Moreover, this $2.8-\mathrm{kb}$ mRNA was clearly inducible by lysoPC with a time-course identical to that of induction of COX-2. This suggests that this $2.8-\mathrm{kb}$ mRNA species is related to COX-2. As we have not characterized this $2.8-\mathrm{kb}$ COX-2-related mRNA, its nature remains unknown. We can speculate, however, that it may be a product of alternative splicing of COX-2 mRNA or a stable product of degradation of 44.5-kb COX-2 mRNA. To the best of our knowledge induction of 2.8-kb COX-2-related mRNA has not been reported. Thus, it is possible that this phenomenon is specific for the induction of COX-2 by lysoPC.

Our nuclear runoff experiments demonstrated that the induction of COX-2 by lysoPC is due to the activation of COX-2 gene. Consistently, with the lack of effect of lysoPC on COX$1 \mathrm{mRNA}$ and protein levels, we did not observe any lysoPCinduced changes of COX-1 transcription rate.

Induction of COX-2 by lysoPC may have profound consequences for the endothelial function, as it resulted in the enhanced synthesis of prostacyclin. Our experiments demonstrated that both the basal as well as calcium ionophore A23187-stimulated synthesis of prostacyclin were increased in HUVEC stimulated with lysoPC. These results, when considered in conjunction with the evidence for the simultaneous induction of COX-2 expression, implicate that COX-2 plays a pivotal role in lysoPCinduced enhancement of prostacyclin synthesis.

Although it is well recognized that COX-2 can be induced in many cell-types, including HUVEC, by cytokines and mitogenic factors, to our knowledge, induction of COX-2 by a lipid mediator such as lysoPC is a novel observation. This finding raises several intriguing questions regarding the biological significance of lysoPC-triggered induction of COX-2 expression, the 
mechanisms involved in lysoPC-triggered activation of the COX-2 gene, and the potential role of lysoPC as an intracellular messenger.

Our findings of the induction of COX-2 but not of COX-1 in HUVEC by lysoPC are in keeping with current concepts regarding the roles of cyclooxygenase isoforms in controlling prostanoid synthesis (7). COX-1 is expressed constitutively in many cell types, including endothelial cells, whereas expression of COX-2 requires stimulation of cells with cytokines, mitogens, or other cell-specific signals. The COX-1 gene is regulated by a tight control mechanism whereas the COX-2 gene is highly inducible. As we have recently demonstrated (29), COX-1 is rapidly degraded during catalysis and, therefore, has only a limited capacity to support elevated synthesis of prostanoids in stimulated endothelial cells. These properties of COX-1 suggest that it plays a major role in the basal synthesis of prostanoids. In contrast, rapid induction of COX-2 is required for augmenting prostanoids synthesis in response to extracellular stimuli.

As outlined in the introduction, lysoPC is believed to play a role in the pathogenesis of atherosclerosis. Several biological actions of lysoPC are consistent with the function of lysoPC as a proatherogenic molecule (17-25). Surprisingly, we have recently shown that lysoPC enhances the endothelial expression of nitric oxide synthase type III (25a) which is believed to be an important antiatherogenic and vasoprotective enzyme. As prostacyclin, the major endothelium-derived prostanoid, is a vasoprotective, antithrombotic, antiplatelet, growth-suppressant molecule (14), the induction of COX-2 may be considered as another example of induction by lysoPC of a vasoprotective and potentially antiatherogenic endothelial gene. However, several reservations have to be made at this point because the role of cyclooxygenases and prostanoids in the pathogenesis of atherogenesis are currently poorly understood (30). Our results are consistent with observations demonstrating that oxidized LDL enhance the endothelial synthesis of prostacyclin $(31,32)$ as well as COX-2 mRNA levels (33). However, the effects of atherosclerosis on prostanoid synthesis in humans or experimental animals have not been conclusively established as increased $(34,35)$, biphasic $(28)$ and reduced prostanoid $(36)$ synthesis have been reported. Similarly, contradictory effects of nonselective inhibitors of $\mathrm{COX}$ on the development of experimental atherosclerosis have been observed (37-39), and the effects of isoform-selective COX inhibitors have not been described yet. We are also unaware of studies investigating cellular distribution of COX-isoforms in human atherosclerotic lesions or animal models of atherosclerosis. Importantly, it is also not known whether lysoPC induces COX-2 in other cellular components of atherosclerotic lesions such as macrophages or vascular smooth muscle cells. In contrast to endothelium, induction of COX-2 in these cells may lead to the production of mainly proinflammatory prostanoids which may contribute to the vascular damage. These limitations preclude any definitive statement about the overall role of induction of COX-2 by lysoPC in the atherogenesis. However, the biological actions of prostacyclin, a major endothelium-derived prostanoid, support the notion that the endothelial expression of COX-2 is a vasoprotective mechanism. It can be conceived that the induction of COX-2 by lysoPC is a back-up mechanism necessary to support elevated synthesis of vasoprotective prostacyclin by stressed endothelial cells. In this context, it is important to note that the vasoprotective and antiatherogenic actions of prostacyclin and nitric oxide significantly overlap and sometimes, as in the case of inhibition of platelet aggregation (40), are synergistic. Therefore, a coordi- nated induction of endothelial enzymes involved in synthesis of nitric oxide and prostacyclin will have a more significant vasoprotective effect than the induction of nitric oxide synthase type III or COX-2 alone. Our findings that lysoPC can induce vasoprotective endothelial genes may seem to disagree with a proposed function of lysoPC as a proatherogenic molecule. However, they can be easily rationalized on grounds of a hypothesis that atherosclerosis begins as a defensive mechanism in response to injury (41). According to this hypothesis, it makes perfect sense that vasoprotective mechanisms are part of an early atherogenic response which is initially designed to serve its defensive function and then regress.

LysoPC has been shown to induce expression of several genes such as vascular adhesion molecule-1, intercellular adhesion molecule-1, PDGF chains A and B, heparin-binding EGF, and nitric oxide synthase-III (23-25). However, in none of the cases have the transduction mechanisms that mediate gene activation by lysoPC been established. Hints as to possible intracellular pathways involved in lysoPC-induced activation of COX-2 gene may be suggested by biological actions of lysoPC in endothelial cells. These include stimulation of protein kinase C activity (42), augmentation of intracellular concentration of $\mathrm{Ca}^{2+}(43)$, and uncoupling of cell surface receptors from $\mathrm{G}$ proteins (44). It is especially likely that the induction of COX2 by lysoPC is at least in part mediated by activation of protein kinase $\mathrm{C}$ because phorbol esters are potent inducers of COX-2 in many cells, including HUVEC (9). However, activation of COX-2 gene by extracellular signals has been shown to depend on many other intracellular signaling pathways involving cAMP (45), tyrosine kinases $(45,46)$, retinoic acid, or platelet activating factor (47). Therefore, further detailed work is required to establish transduction mechanism of activation of COX-2 by lysoPC. Interestingly, significant amounts of lysoPC can be generated intracellularly upon the action of phospholipase $A_{2}$ on membrane phosphatidylcholine, the most abundant phospholipid. LysoPC has been recently suggested as an intracellular mediator of protein kinase $\mathrm{C}$ activation after receptor stimulation (48). Therefore, a possibility exists that lysoPC serves as an intracellular messenger involved in the regulation of gene expression. In this context, it may be noted that in many cells induction of COX-2 and phospholipase $A_{2}$ occur simultaneously $(49,50)$.

We conclude that lysoPC induces expression of COX-2 in human umbilical vein endothelial cells by a transcriptional mechanism. This results in the enhancement of endothelial synthesis of prostacyclin. These findings strongly support our previous suggestion that lysoPC may be an important signal molecule involved in the regulation of expression of vasoprotective endothelial genes.

\section{Acknowledgments}

We thank Dr. Richard Kulmacz (Division of Hematology, University of Texas Houston Health Science Center) for COX-1 and COX-2 protein standards, anti-COX-1 antibody, and review of the manuscript, Dr. Timothy Hla for COX-2 cDNA, and Dr. Paul Marshall for anti-COX2 antibody.

This work was supported by National Institutes of Health grants RO1 HL-53223 and NS-23327.

\section{References}

1. Smith, W. L., and L. J. Marnett. 1991. Prostaglandin endoperoxide synthase: structure and catalysis. Biochim. Biophys. Acta. 1083:1-17. 
2. Hemler, M., W. E. M. Lands, and W. L. Smith. 1976. Purification of the cyclooxygenase that forms prostaglandins. Demonstration of two forms of iron in the holoenzyme. J. Biol. Chem. 261:5575-5579.

3. DeWitt, D. L., and W. L. Smith. 1988. Primary structure of prostaglandin $\mathrm{G} / \mathrm{H}$ synthase from sheep vesicular gland determined from the complementary DNA sequence. J. Biol. Chem. 85:1412-1416.

4. Merlie, J. P., D. Fagan, J. Mudd, and P. Needleman. 1988. Isolation and characterization of the complementary DNA for sheep seminal vesicle prostaglandin endoperoxide synthase (cyclooxygenase). J. Biol. Chem. 263:3550-3553.

5. Kujubu, D. A., B. S. Fletcher, B. C. Varnum, R. W. Lim, and H. R. Herschman. 1991. TIS10, a phorbol ester tumor promoter-inducible mRNA from Swiss 3T3 cells, encodes a novel prostaglandin synthase/cyclooxygenase homologue. J. Biol. Chem. 266:12866-12872.

6. Fletcher, B. S., D. A. Kujubu, D. M. Perrin, and H. R. Herschman. 1992. Structure of the mitogen-inducible TIS10 gene and demonstration that the TIS10encoded protein is a functional prostaglandin G/H synthase. J. Biol. Chem. 267:4338-4344.

7. Herschman, H. R. 1994. Regulation of prostaglandin synthase-1 and prostaglandin synthase-2. Cancer Metastasis Rev. 13:241-256.

8. DeWitt, D. L. 1991. Prostaglandin endoperoxide synthase: regulation of enzyme expression. Biochim. Biophys. Acta. 1083:121-134.

9. Hla, T., and K. Neilson. 1992. Human cyclooxygenase-2 cDNA. Proc. Natl. Acad. Sci. USA. 89:7384-7388.

10. Jones, D. A., D. P. Carlton, T. M. McIntyre, G. A. Zimmerman, and S. M. Prescott. 1993. Molecular cloning of human prostaglandin endoperoxide synthase type II and demonstration of expression in response to cytokines. J. Biol. Chem. 268:9049-9054.

11. Wu, K. K., R. J. Kulmacz, L. Wang, D. Loose-Mitchell, and A. Tsai. 1992. Molecular biology of prostacyclin biosynthesis. In Prostacylin: New Perspectives for Basic Research and Novel Therapies. G. M. Rubanyi and J. R. Vane, editors. Elsevier Science Publishers B. V., Amsterdam. 11-23.

12. Diaz, A., A. M. Reginato, and S. Jimenez. 1991. Alternative splicing of human prostaglandin $\mathrm{G} / \mathrm{H}$ synthase mRNA and evidence of differential regulation of the resulting transcripts by transforming growth factor betal, interleukin lbeta, and tumor necrosis factor alpha. J. Biol. Chem. 267:10816-10822.

13. Moncada, S., R. J. Gryglewski, S. Bunting, and J. R. Vane. 1976. An enzyme isolated from arteries transforms prostaglandin endoperoxides to an unstable substance that inhibits platelet aggregation. Nature (Lond.). 263:663-665.

14. Vane, J. R., and R. M. Botting. 1995. Pharmacodynamic profile of prostacyclin. Am. J. Cardiol. 75:3A-10A.

15. Szczeklik, A., R. Nizankowski, S. Slawinski, G. Szczeklik, P. Gluszko, and R. J. Gryglewski. 1979. Succesful therapy of advanced atherosclerosis obliterans with prostacyclin. Lancet. i:1111-1114.

16. Szczeklik, A., and R. J. Gryglewski. 1981. Treatment of vascular disease with prostacyclin. In Clinical Pharmacology of Prostacyclin. P. J. Lewis and J. O'Grady, editors. Raven Press, Ltd., New York. 159-167.

17. Vidaver, G. A., A. Ting, and J. W. Lee. 1985. Evidence that lysolecithin is an important causal agent of atherosclerosis. J. Theor. Biol. 115:27-41.

18. Portman, O. W., and M. Alexander. 1969. Lysophosphatidylcholine concentrations and metabolism in aortic intima plus inner media: effect of nutritionally induced atherosclerosis. J. Lipid Res. 10:158-165.

19. Parthasarathy, S., U. P. Steinbrecher, J. Barnett, J. L. Witztum, and D. Steinberg. 1985. Essential role of phospholipase A2 activity in endothelial cellinduced modification of low density lipoprotein. Proc. Natl. Acad. Sci. USA 82:3000-3004.

20. Quinn, M. T., S. Parthasarathy, and D. Steinberg. 1988. Lysophosphatidylcholine: a chemotactic factor for human monocytes and its potential role in atherogenesis. Proc. Natl. Acad. Sci. USA. 85:2805-2809.

21. Parthasarathy, S., M. T. Quinn, D. C. Schwenke, T. E. Carew, and D. Steinberg. 1989. Oxidative modification of beta-very low density lipoprotein. Potential role in monocyte recruitment and foam cell formation. Arteriosclerosis. 9:398-404

22. Sakai, M., A. Miyazaki, H. Hakamata, T. Sasaki, S. Yui, M. Yamazaki, M. Schichiri, and S. Horiuchi. 1994. Lysophosphatidylcholine plays an essentia role in the mitogenic effect of oxidized low density lipoprotein on murine macrophages. J. Biol. Chem. 269:31430-31435.

23. Nakano, T., E. W. Raines, J. A. Abraham, M. Klagsbrun, and R. Ross. 1994. Lysophosphatidylcholine upregulates the level of heparin-binding epiderma growth factor-like growth factor mRNA in human monocytes. Proc. Natl. Acad. Sci. USA. 91:1069-1073.

24. Kume, N., M. I. Cybulsky, and M. A. Gimbrone, Jr. 1992. Lysophosphatidylcholine, a component of atherogenic lipoproteins, induces mononuclear leukocyte adhesion molecules in cultured human and rabbit arterial endothelial cells. J. Clin. Invest. 90:1138-1144.

25. Kume, N., and M. A. Gimbrone, Jr. 1994. Lysophosphatidylcholine transcriptionally induces growth factor gene expression in cultured human endothelia cells. J. Clin. Invest. 93:907-911.

25a. Zembowicz, A., J.-L. Tang, and K. K. Wu. 1995. Transcriptional induc- tion of endothelial nitric oxide synthase type-III by lysophosphatidylcholine. $J$. Biol. Chem. 270:17006-17010.

26. Wu, K. K., H. Hatzakis, S. S. Lo, D. C. Seong, S. K. Sanduja, and H. H. Tai. 1988. Stimulation of de novo synthesis of prostaglandin G/H synthase in human endothelial cells by phorbol ester. J. Biol. Chem. 263:19043-19047.

27. Greenberg, M. E., and T. P. Bender. 1992. Short Protocols in Molecular

Biology. F. M. E. Ausubel, editor. Greene Publishing Associates and John Wiley \& Sons, Inc., New York. 4-25-4-29.

28. Salmon, J. A. 1978. A radioimmunoassay for 6-keto-prostaglandin $F_{2 \alpha}$. Prostaglandins. 15:383-397.

29. Sanduja, S. K., A. L. Tsai, N. Matijevic-Aleksic, and K. K. Wu. 1994 Kinetics of prostacyclin synthesis in PGHS-1-overexpressed endothelial cells. Am. J. Physiol. 267:C1459-C1466.

30. Pomerantz, K. B., and D. P. Hajiar. 1989. Eicosanoids in regulation of arterial smooth muscle cell phenotype, proliferative capacity, and cholesterol metabolism. Arteriosclerosis. 9:413-429.

31. Holland, J. A., K. A. Pritchard, N. J. Rogers, and M. B. Stemerman. 1988 Perturbation of cultured human endothelial cells by atherogenic levels of low density lipoprotein. Am. J. Pathol. 132:474-478.

32. Triau, J. E., S. N. Meydani, and E. J. Schaefer. 1988. Oxidized low density lipoprotein stimulates prostacyclin production by adult human vascular endothelia cells. Arteriosclerosis. 8:810-818.

33. Maier, J. A., L. Barenghi, S. Bradamante, and F. Pagani. 1994. Modulators of oxidized LDL-induced hyperadhesiveness in human endothelial cells. Biochem. Biophys. Res. Commun. 204:673-677.

34. FitzGerald, G. A., B. Smith, A. K. Pedersen, and A. R. Brush 1984 Increased prostacyclin biosynthesis in patients with severe atherosclerosis and platelet activation. N. Engl. J. Med. 310:1065-1068.

35. Mehta, J. L., D. Lawson, P. Mehta, and T. Saldeen. 1988. Increased prostacyclin and thromboxane A2 biosynthesis in atherosclerosis. Proc. Natl. Acad. Sci. USA. 85:4511-4515.

36. Rolland, P., R. Jouve, E. Pellegrin, C. Mercier, and A. Serradimigni. 1984. Alteration in prostacyclin and prostaglandin E2 production. Correlation with changes in human atherosclerotic disease. Arteriosclerosis. 4:70-78.

37. Sun, Y. P., B. Q. Zhu, R. E. Sievers, W. M. Isenberg, and W. W. Parmley. 1993. Aspirin inhibits platelet activity but does not attenuate experimental atherosclerosis. Am. Heart J. 125:79-86.

38. Debons, A. F., K. Fani, and F. A. Jimenez. 1981. Enhancement of experimental atherosclerosis by aspirin. J. Toxicol. Environ. Health. 8:899-906.

39. Stoller, D. K., C. B. Grorud, V. Michalek, and H. Buchwald. 1993. Reduction of atherosclerosis with nonsteroidal anti-inflammatory drugs. J. Surg. Res. 54:7-11.

40. Radomski, M. W., R. M. J. Palmer, and S. Moncada. 1987. The antiaggregating properties of vascular endothelium: interactions between prostacyclin and nitric oxide. Br. J. Pharmacol. 92:639-646.

41. Ross, R. 1993. Atherosclerosis: a defense mechanism gone awry, Am. J. Pathol. 143:987-1002.

42. Kugiyama, K., M. Ohgushi, S. Sugiyama, T. Murohara, K. Fukunaga, E Miyamoto, and H. Yasue. 1992. Lysophosphatidylcholine inhibits surface receptor-mediated intracellular signals in endothelial cells by a pathway involving protein kinase C activation. Circ. Res. 71:1422-1428. (Published erratum appears in Circ. Res. 72:723).

43. Inoue, N., K. Hirata M. Yamada, Y. Hamamori, Y Matsuda, H. Akita, and M. Yokoyama. 1992. Lysophosphatidylcholine inhibits bradykinin-induced phosphoinositide hydrolysis and calcium transients in cultured bovine aortic endothelial cells. Circ. Res. 71:1410-1421.

44. Flavahan, N. A. 1993. Lysophosphatidylcholine modifies $G$ protein-dependent signaling in porcine endothelial cells. Am. J. Physiol. 264:H722-H727.

45. Morris, J. K., and J. S. Richards. 1993. Hormone induction of luteinization and prostaglandin endoperoxide synthase- 2 involves multiple cellular signaling pathways. Endocrinology. 133:770-779.

46. Kester, M., E. Coroneos, P. J. Thomas, and M. J. Dunn. 1994. Endothelin stimulates prostaglandin endoperoxide synthase- 2 mRNA expression and protein synthesis through a tyrosine kinase-signaling pathway in rat mesangial cells. $J$. Biol. Chem. 269:22574-22580.

47. Bazan, N. G., B. S. Fletcher, H. R. Herschman, and P. K. Mukherjee. 1994. Platelet-activating factor and retinoic acid synergistically activate the inducible prostaglandin synthase gene. Proc. Natl. Acad. Sci. USA. 91:5252-5256.

48. Nishizuka, Y. 1992. Intracellular signaling by hydrolysis of phospholipids and activation of protein kinase C. Science (Wash. DC). 258:607-614.

49. Roshak, A., G. Sathe, and L. A. Marshall. 1994. Suppression of monocyte 85-kDa phospholipase A2 by antisense and effects on endotoxin-induced prostaglandin biosynthesis. J. Biol. Chem. 269:25999-26005.

50. Hulkower, K. I., S. J. Wertheimer, W. Levin, J. W. Coffey, C. M. Anderson, T. Chen, D. L. DeWitt, R. M. Crowl, W. C. Hope, and D. W. Morgan 1994. Interleukin-1 beta induces cytosolic phospholipase A2 and prostaglandin H synthase in rheumatoid synovial fibroblasts. Evidence for their roles in the production of prostaglandin E2. Arthritis Rheum. 37:653-661. 